Research Article

\title{
Formulation and Evaluation of Nanosuspension as an Alternative Approach for Solubility Enhancement of Simvastatin
}

\author{
Mohini E. Shinde*, Mitesh P. Sonawane, Avish D. Maru \\ Department of Pharmaceutics, Loknete Dr. J. D. Pawar College of Pharmacy Manur, Kalwan, Maharashtra, India \\ *Corresponding author's E-mail: shindemohini494@gmail.com
}

Received: 14-08-2021; Revised: 21-10-2021; Accepted: 28-10-2021; Published on: 15-11-2021. \begin{abstract}
Solubility is an essential factor for drug effectiveness. Simvastatin is poorly water-soluble drug and its bioavailability is very low. Nanosuspension is one of those approach which can tremendously enhance the effective surface area of drug particles by reducing the particle size and there by increases the rate of dissolution and hence improve bioavailability. The main purpose of the present investigation was to increase the saturation solubility of simvastatin by preparation of nanosuspension. Nanosuspension of simvastatin were prepared by nanoprecipitation method using hydroxypropyl cellulose as stabilizer and sodium lauryl sulphate as surfactant. Prepared nanosuspension was evaluated for its particle size, total drug content, entrapment efficiency and saturation solubility study. On the basis of the evaluation, the best batch F8 formulation demonstrated highest drug content and entrapment efficiency with average particle size of $0.004 \mu \mathrm{m}$. The saturation solubility studies show the solubility of the prepared nanosuspension has increased as compared to the pure drug due to the particle size reduction. The nanosuspension of simvastatin could be successfully prepared and can be concluded that the nanosuspension formulation is a promising approach to enhance the solubility. The nanoprecipitation is a simple and effective method to produce nano sized particles of poorly water-soluble drugs with enhance solubility.
\end{abstract}

Keywords: Simvastatin, Nanosuspension, Saturation solubility, Particle size, Dissolution, Bioavailability, Nanoprecipitation.

QUICK RESPONSE CODE $\rightarrow$

DOI:

10.47583/ijpsrr.2021.v71i01.012

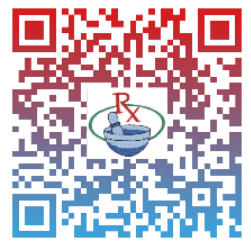

DOI link: http://dx.doi.org/10.47583/ijpsrr.2021.v71i01.012

\section{INTRODUCTION}

S

olubility is a vital factor for developing drug delivery systems for hydrophobic drugs. One of the major problems associated with poorly soluble drugs is very low bioavailability ${ }^{1}$. This problem is more worsen because of more than the $40 \%$ of the newly discovered drug product are poorly water soluble. BCS Class II and IV Drugs are poorly water soluble and their pharmacokinetic studies shows low oral bioavailability ${ }^{2}$. So, they show problems in formulating them in conventional dosage forms. Formulation of these mainly the BCS class II and IV drug into the conventional dosage form is a challenging problem faced by the pharmaceutical researcher. In this cases, preparing nanosuspension is preferred for such compounds that are insoluble in water but soluble in oil ${ }^{3}$. Nanosuspension is one those approach used to solve the problems of poor solubility which is based on size reduction mechanism. Reduction of drug particles in nanosized range leads to enhance faster dissolution rate and increased surface area ${ }^{4}$. Recently, nanoscale systems have received much interest as a way to resolve solubility issues because of their technical simplicity compared to liposomes and other colloidal drug carriers. Nanosuspensions have proven to be a better alternatives over other approaches currently available for improving solubility of number of drugs ${ }^{5}$. Nanosuspension consist of dispersed solid drug particles in an aqueous vehicle with average particle sizes below $1 \mu \mathrm{m}$. they are stabilized by polymers and surfactants. The potential advantage of nanosuspension technology is to enhance the saturation solubility and consequently increase the dissolution rate of the drug ${ }^{6,7}$.

Simvastatin is a hydrophobic and highly lipophilic drug belonging to the BCS Class II drug, the dissolution process of this drug acts as the rate controlling step and therefore it is necessary to improve the solubility ${ }^{8}$. Simvastatin is a lipid lowering agent shows $5 \%$ bioavailability due to poor solubility, decreased in bioavailability due to rapid first pass metabolism. Approximately $95 \%$ of an oral dose is not absorbed. Therefore, it is important to enhance the aqueous solubility and improving bioavailability. Simvastatin is a potent inhibitor of HMG-COA Reductase which is rate limiting step in cholesterol biosynthesis ${ }^{9,10}$.

The main intention of this research is to enhance the aqueous solubility and therefore improved the bioavailability of poorly aqueous soluble drug like simvastatin. Formulation as simvastatin nanosuspension is an attractive and promising alternatives to solve these problems. Improvement of aqueous solubility in such case is a valuable goal to improve therapeutic efficacy. 


\section{MATERIALS AND METHODS}

\section{Materials}

Simvastatin was acquired as a gift sample from Artemis Biotech, Hyderabad. Hydroxy propyl cellulose and Sodium lauryl sulphate were procured from Research Lab Mumbai, whereas Methanol was purchased from Fischer Scientific, Mumbai. All the ingredients used in the research work were of analytical grade.

\section{Preparation of simvastatin nanosuspension}

The nanoprecipitation technique is used for the preparation of simvastatin nanosuspension ${ }^{11}$. The pure drug of simvastatin $(10 \mathrm{mg})$ was dissolve in $(1 \mathrm{ml})$ of methanol to produced uniform organic phase. (Solution 1) The Stabilizers (HPC, SLS) was dissolved in water $(40 \mathrm{ml})$ to form aqueous phase. (solution 2) The solution 1 added into solution 2 dropwised with the help of syringe under the magnetic stirrer for $15 \mathrm{~min}$. to get desired nano dispersion. After the magnetic stirring the formulation is subjected to agitation with the help of lab stirrer at $1500 \mathrm{rpm}$ at $1 \mathrm{hr}$. to evaporate the organic solvent.

Table 1: Formulation batches of Simvastatin Nanosuspension.

\begin{tabular}{|c|c|c|c|c|c|c|c|c|c|}
\hline Ingredients & \multicolumn{8}{|c|}{ Formulation Code } \\
\hline Simvastatin (mg) & F1 & F2 & F3 & F4 & F5 & F6 & F7 & F8 & F9 \\
\hline HPC (\%) & 10 & 10 & 10 & 10 & 10 & 10 & 10 & 10 & 10 \\
\hline SLS (\%) & 1 & 2.5 & 5 & - & - & - & 1 & 2.5 & 5 \\
\hline Methanol (ml) & - & - & - & 0.1 & 0.3 & 0.5 & 0.1 & 0.3 & 0.5 \\
\hline Water (ml) & 1 & 1 & 1 & 1 & 1 & 1 & 1 & 1 & 1 \\
\hline Stirring Speed (RPM) & 40 & 40 & 40 & 40 & 40 & 40 & 40 & 40 & 40 \\
\hline
\end{tabular}

\section{Particle size analysis}

The average particle size of all NS formulations was determined by the motic digital microscopy. The nanosuspension showing the lowest particle size was selected for further studies. The particle size of the formulated batches was measured in micrometers ${ }^{12}$.

\section{Total drug content}

Prepared Nanosuspensions was analyzed for drug content by UV spectroscopic method. Different batches of nanosuspensions equivalent to $10 \mathrm{mg}$ of drug Simvastatin weighed accurately and diluted up to $100 \mathrm{ml}$ with methanol. Stock solutions will be diluted with methanol and analyze by UV spectroscopy at $238 \mathrm{~nm}^{13}$.

\section{Entrapment efficiency}

The method suitable for determining entrapment efficiency of nanosuspension when fairly high concentration of free drug is present in the supernatant after centrifugation ${ }^{14,15}$. $10 \mathrm{ml}$ portion of the freshly prepared nanosuspension was centrifuged at $1000 \mathrm{rpm}$ for $10 \mathrm{~min}$. using centrifuge. the supernatant was removed and the amount of incorporated drug was measured by taking the absorbance of supernatant solution at $238 \mathrm{~nm}$ by using UV spectrophotometer. (LABINDIA $3000^{+}$).

Entrapment efficiency was calculated by following formula:

\section{Entrapment Efficiency (\%) =}

(W initial drug $-W$ free drug / W initial drug $\times 100$

\section{Saturation solubility studies}

The saturation solubility studies were carried out for both the unprocessed pure drug and its nanosuspension ${ }^{16,17}$. The solubility of simvastatin in powder form was determined by a shake flask method. Briefly, an excess amount of drug simvastatin was suspended in $10 \mathrm{ml}$ of water, and the suspension were shaken and filter through a $0.22 \mu \mathrm{m}$ whatman filter. The filtered solution was suitably diluted and the simvastatin concentration in the filtrate was analysed by UV analysis method at $238 \mathrm{~nm}$. The solubility of best batch of formulation was measured by centrifugal method. Briefly, $10 \mathrm{ml}$ of nanosuspension was loaded into centrifugal tubes. Samples were centrifuged at $10000 \mathrm{rpm}$ for $10 \mathrm{~min}$. The supernatant solutions were analyzed using UV spectrophotometer at $238 \mathrm{~nm}$.

\section{Stability studies}

The final formulation was subjected to stability studies as per ICH guidelines. Samples were stored at $40^{\circ} \mathrm{C} \pm 2{ }^{\circ} \mathrm{C}$ and $75 \% \pm 5 \% \mathrm{RH}$ for 1 month to access their stability. The Various parameters such as drug content, entrapment efficiency was measured before and after 30 days of stability.

\section{RESULTS AND DISCUSSION}

\section{Particle size analysis}

The particle size of the NS produced systems was analysed by motic digital microscopy. the particle size of each formulation was carried out and results indicates that all formulations was found in the nanosized range. The F8 formulation shows better results because of low particle 
size compared to other formulations. The batch F8 had a average particle size of $0.004 \mu \mathrm{m}$ which indicate the particles are in uniform distribution. The best formulation is select on the basis of particle size. The particle size of all formulations as shown in Table 2.

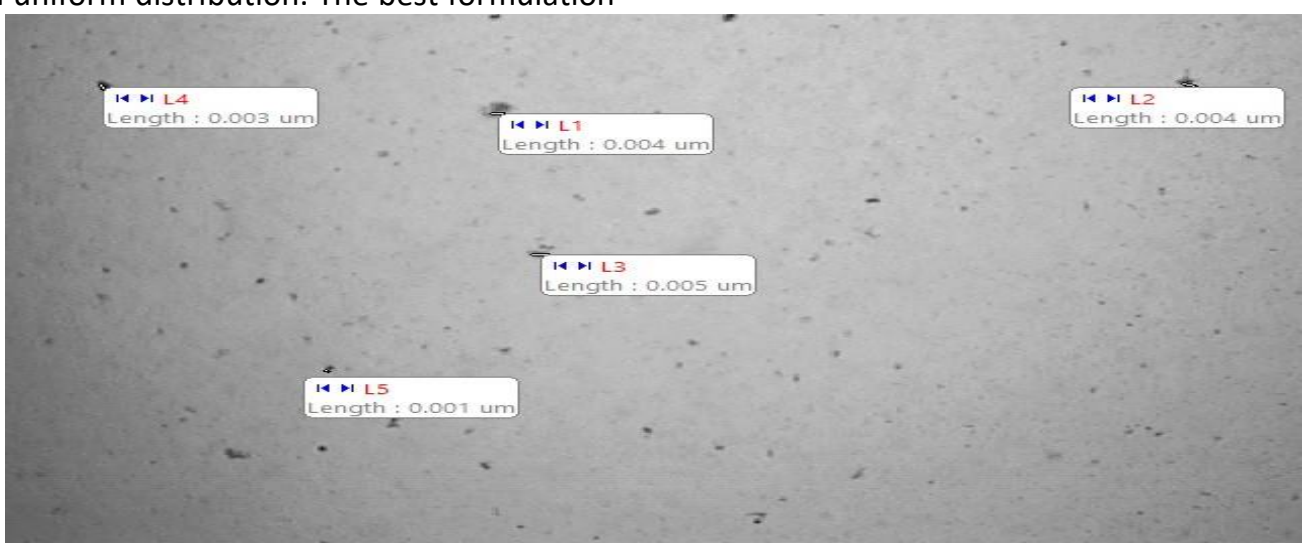

Figure 1: Particle size analysis by motic microscope of F8 Formulation.

Table 2: Particle size, total drug content and entrapment efficiency data of all formulations.

\begin{tabular}{|c|c|c|c|}
\hline Formulation Code & Particle size $(\boldsymbol{\mu m})$ & Total drug content (\%) & Entrapment efficiency (\%) \\
\hline F1 & 0.013 & 83.64 & 81.69 \\
\hline F2 & 0.015 & 85.79 & 83.98 \\
\hline F3 & 0.018 & 83.96 & 81.33 \\
\hline F4 & 0.016 & 85.23 & 84.20 \\
\hline F5 & 0.014 & 87.79 & 85.46 \\
\hline F6 & 0.017 & 81.64 & 79.11 \\
\hline F7 & 0.006 & 91.54 & 87.44 \\
\hline F8 & 0.004 & 95.19 & 93.28 \\
\hline F9 & 0.007 & 93.24 & 90.88 \\
\hline
\end{tabular}

\section{Total drug content}

The drug content of all NS formulations was found to be greater than $81 \%$. Indicating suitability of these methods for particle size reduction. the Formulation batch F8 shows drug content $95.19 \%$. The total drug content of all formulations are recorded in Table 2.

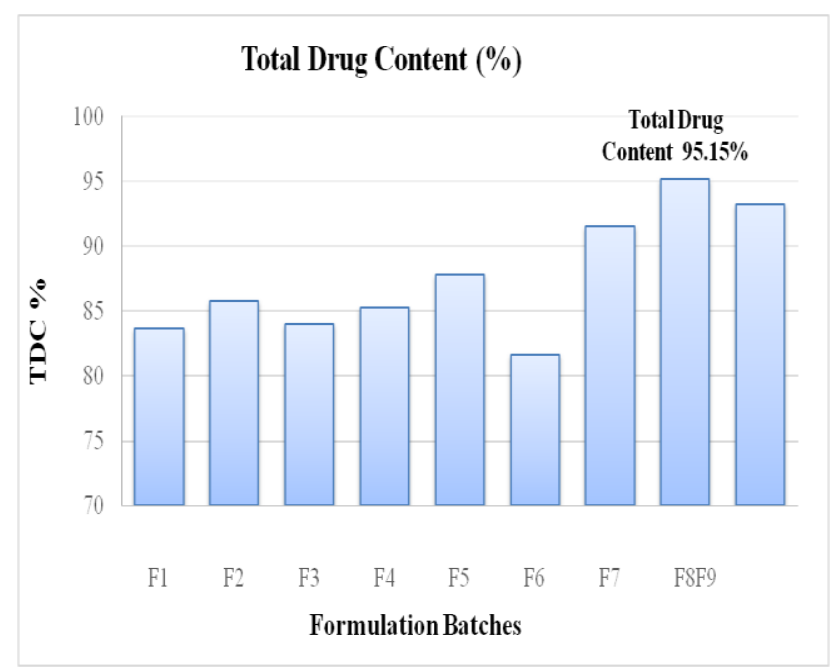

Figure 2: Total drug content of formulated nanosuspensions.

\section{Entrapment efficiency}

The entrapment efficiency of all formulations was found to be in the range of (79.11- 93.28\%) the entrapment efficiency of formulation F8 was high when compared to other formulations. The entrapment efficiency of all nanosuspensions are recorded in Table 2.

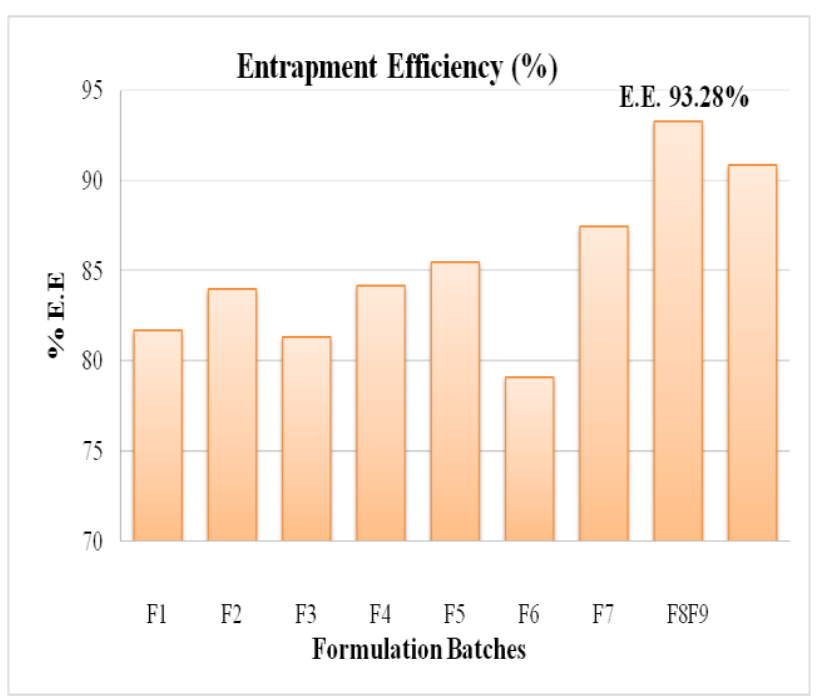

Figure 3: Entrapment efficiency of formulated nanosuspensions. 


\section{Saturation solubility studies}

The saturation solubility studies indicating that nanosuspension showing maximum solubility compared to unprocessed drug. the optimized batch F8 shows highest solubility than pure drug. It may be observed that solubility of prepared nanosuspension has been increased due to the formation stabilized nanoparticles. It may be due to decreased in particle size and increased solubilization.

Table 3: Saturation Solubility of Simvastatin and its nanosuspension.

\begin{tabular}{|c|c|}
\hline Formulations & Saturation solubility $(\mu \mathrm{g} / \mathrm{ml})$ \\
\hline Pure drug & 12.60 \\
\hline Nanosuspension & 64.74 \\
\hline
\end{tabular}

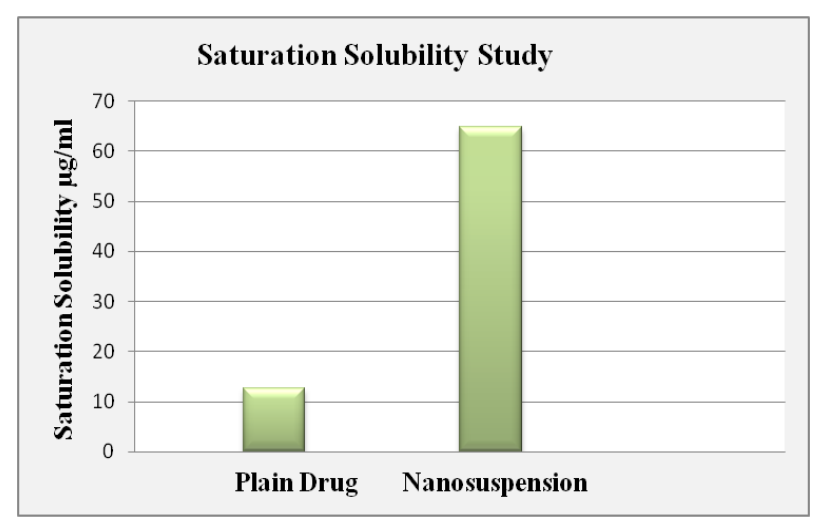

Figure 4: Comparison of solubility of Simvastatin and its Nanosuspension.

It may be observed that the solubility of prepared simvastatin nanosuspension has been increased up to 5.12 fold due to formation of stabilized nanoparticles.

\section{Stability studies}

The stability studies were carried out on final formulation. The samples were stored at $40^{\circ} \mathrm{C} \pm 2{ }^{\circ} \mathrm{C}$ and $75 \% \pm 5 \% \mathrm{RH}$ for 30 days to access best formulation their stability. After 30 days were withdrawn and rested for entrapment efficiency and total drug content. The F8 formulation did not show any significant difference in both parameters. It indicates that this formulation was able to retain its stability up to 30 days.

Table 4: Stability data of F8 formulation of nanosuspension.

\begin{tabular}{|c|c|c|}
\hline Time period & $\begin{array}{c}\text { Entrapment } \\
\text { efficiency }\end{array}$ & $\begin{array}{c}\text { Total drug } \\
\text { content }\end{array}$ \\
\hline Initial (Odays) & $93.28 \%$ & $95.19 \%$ \\
\hline & After storage & \\
\hline 1 month & $92.65 \%$ & $95.02 \%$ \\
\hline
\end{tabular}

It may be observed that the solubility of prepared simvastatin nanosuspension has been increased up to 5.12 fold due to formation of stabilized nanoparticles.

\section{CONCLUSION}

The above investigation suggests the suitability of simvastatin nanosuspension as a promising drug delivery system. Simvastatin nanosuspension was successfully prepared by a nanoprecipitation method with a lower particle size. The comparison study of the simvastatin nanosuspension with pure drug (simvastatin) also indicate that the solubility of nanosuspension is higher than the pure drug. Hence, it can be good alternative to the solubility enhancement. In conclusion, the appropriate selection of process parameter and formulation parameters we can conclude that nanoprecipitation method is a simple and effective approach to produce nanosized particles of poorly water-soluble drugs with enhance solubility. It can be concluded that formulating poorly water-soluble drugs in the form of nanosuspension would be a promising approach in the delivery of poorly water-soluble drug.

\section{REFERENCES}

1. Patel HM, Patel BB, Shah CN. Nanosuspension: a novel approach to enhance solubility of poorly water-soluble drugs- A review. International journal of advances in pharmaceutics. 2016 Mar 20; 5(2): 21-19.

2. Deshkar SS, Sonkamble KG, Mahore JG. Formulation and Optimization of Nanosuspension for Improving Solubility and Dissolution of Gemfibrozil. Asian J Pharm Clin Res. 2019; 12 (1): 157-163.

3. Patel $M$, Shah A, Patel NM, Patel MR, Patel KR. Nanosuspension: A novel approach for drug delivery system. Jpsbr. 2011; 1(1): 1-10.

4. Lakshmi P, Kumar GA. Nanosuspension technology: A review. Int J Pharm Sci. 2010 Aug; 2(4): 35-40.

5. Thattil JG, Kumar KK, Kumar DB. Nanosuspension technology in pharmaceuticals. J.Bio. Innov 2018; 7(2): 660-677.

6. Jiraporn C. Nanosuspension Technology for Drug Delivery, Walailak J Sci \& Tech. 2007; 4(2): 139-153.

7. Shid RL, Dhole SN, Kulkarni N, Shid SL. Nanosuspension: a review. Int J Pharm Sci Rev Res. 2013 Sep; 22(1): 98-106.

8. Shid RL, Dhole SN, Kulkarni N, Shid SL. Formulation and evaluation of nanosuspension delivery system for simvastatin. Int J Pharm Sci and Nanotechnol. 2014 May 31; 7: 2459- 76

9. CAUSEVIC-RAMOSEVAC AN, Semiz S. Drug interactions with statins. Acta Pharmaceutica. 2013 Sep 30; 63(3): 277293.

10. Raturi A, Bhatt G, Kothiyal P. formulation and evaluation of nanosuspension of Rosuvastatin calcium, Int J Drug Reg Affairs. 2013; 1(3): 14-18.

11. Sundar V, Divya P, Sridevi P, Akhila K, Dhanaraju M. Design, Formulation and Evaluation of Nanosuspension for Drug Delivery of Celecoxib. International Journal of Pharmaceutical Research, 11, 2019.

12. Patil AM, Patil IN, Mane RU, Randive DS, Bhutkar MA, Bhinge SD. Formulation optimization and evaluation of 
cefdinir nanosuspension using factorial design. Marmara Pharm J., 2018; 22: 587-98.

13. Patel HM, Patel UB, Shah C, Akbari B. Formulation and Development of Nanosuspension as an Alternative Approach for Solubility and Dissolution Enhancement of Aceclofenac. International Journal of Advances in Pharmaceutics, 2018; 33-47.

14. Chirumamilla SK, Padasala UD, Aravally H, Vuppalapati L, Cherukuri S. Solubility and Dissolution enhancement of meropenem by nanosuspension approach, J Young Pharm. 2017; 9(3): 429-435.

15. Noor MD, Shaimaa N, Abdul H, Ahmed AH. Formulation and characterization of lafutidine nanosuspension for oral drug delivery system, Int J App pharm. 2018; 12(2): 20-30

16. Nayak S, Panda D, Patnaik AK. Formulation Design And In Vitro Characterization of Felodipine Nano-Suspension.
International Journal of Pharmaceutical Sciences and Research. 2015 Jun 1; 6(6): 2587.

17. Papdiwal AP, Pande VV, Aher S. Investigation of effect of different stabilizers on formulation of zaltoprofen nanosuspension. Int J Pharm Sci Rev Res. 2014 Jul; 27(2): 244-9.

18. Nerker PP, Mahajan H, Deore S, Ige P. Formulation and Evaluation of Nanosuspension Formulations of Ramipril using Hydrophilic Polymers. International Journal of Pharmaceutical Sciences and Nanotechnology. 2015 Feb 28; 8(1): 2735-41.

19. Maddukuri S, Srawanthi P, Metta S, Radha GV, Krishna KS M. Screening of Stabilizers in Azithromycin nanosuspensions. Int. J. Pharm. Sci. Rev. Res., 2019; 55: 7784 .

Source of Support: The author(s) received no financial support for the research, authorship, and/or publication of this article.

Conflict of Interest: The author(s) declared no potential conflicts of interest with respect to the research, authorship, and/or publication of this article.

For any question relates to this article, please reach us at: editor@globalresearchonline.net New manuscripts for publication can be submitted at: submit@globalresearchonline.net and submit_ijpsrr@rediffmail.com 